\title{
Agent Technology and Wireless Sensor Networks for Monitoring Patients in Residences and Their Homes
}

\author{
Ricardo S. Alonso, Dante I. Tapia, Gabriel Villarrubia, and Juan F. De Paz \\ Departamento Informática y Automática Universidad de Salamanca \\ Plaza de la Merced s/n, 37008, Salamanca, Spain \\ \{ralorin, dantetapia,gvg, fcofds\} @usal.es
}

\begin{abstract}
This paper presents an intelligent multi-agent system aimed at improving healthcare and assistance to elderly and dependent people in geriatric residences and their homes. The system is based on the PANGEA and HERA platforms and integrates a set of autonomous reactive and deliberative agents designed to support the carers' activities and to guarantee that the patients are given the right care. The system makes use of Wireless Sensor Networks and a Real-Time Locating System for providing autonomous responses according to the environment status. A case study where the multi-agent system has been tested in several real environments is presented.
\end{abstract}

Keywords: multi-agent systems, deliberative agents, real-time locating systems, wireless sensor networks, health care.

\section{Introduction}

The importance of developing new and more reliable ways to provide care and support to people is underlined by the ageing of world population, especially in industrialized countries [1], and the creation of secure, unobtrusive and adaptable environments for monitoring and optimizing health care will become vital. Therefore, tomorrow health care institutions (public or private) will be equipped with intelligent systems capable of interacting with humans.

Research areas such as Ambient Intelligence (AmI) and Ambient Assisted Living (AAL) propose news way to interact between people and technology, where this last one is adapted to individuals and their context [2]. The objective of AmI and AAL systems is to develop intelligent and intuitive systems and interfaces capable to recognize and respond to the user's necessities in a ubiquitous way, providing capabilities for ubiquitous computation and communication, considering people in the center of the development, and creating technologically complex environments in different fields, such as medical, domestic or academic, among many others.

On the one hand, Agent Technology [3] have become increasingly relevant for developing distributed and dynamic intelligent environments and therefore fulfils the requirements and goals of Ambient Intelligence and Ambient Assisted Living. These intelligent systems aim to support people in several aspects of their daily life, 
predicting potential hazardous situations and delivering physical and cognitive support. In this sense, it is important to integrate intelligent and dynamic mechanisms to learn from past experiences and therefore provide users with better tools for supplying healthcare.

On the other hand, Wireless Sensor Networks (WSNs) allow us to obtain information about the environment and act on this, expanding users' capabilities and automating daily actions [4]. One of the most interesting applications for WSNs is Real-Time Locating Systems (RTLS). Although outdoor locating is well covered by systems such as the current GPS (Global Positioning System), indoor locating needs still more development, especially with respect to accuracy and low-cost and efficient infrastructures [5]. In this sense, the use of optimized locating techniques allows obtaining more accurate locations using even fewer sensors and with less computational requirements [5].

This paper presents an intelligent multi-agent system aimed at improving healthcare and assistance to elderly and dependent people in geriatric residences and their homes. The system is based on the PANGEA (Platform for Automatic coNstruction of orGanizations of intElligents Agents) [6] and HERA (HardwareEmbedded Reactive Agents) platforms designed and developed by the BISITE research group [7], as an experimental deployment in a real scenario. The system integrates a set of autonomous reactive and deliberative agents designed to support the carers' activities and to guarantee that the patients are given the right care in both the residence and their homes. The system is divided into two sub-systems: the first one is running in the geriatric residence and acts as a control center; and the second one is running at the patient's home and is always linked to the first one. The system incorporates autonomous agents that manage information gathered from a wireless sensor network and a real-time locating system to provide the system with innovative interaction schemas using mobile devices.

The next section describes the motivation and explains why there is a need for developing a new telemonitoring system. Then, the system's main features and components are briefly described, including its architecture, as well as the PANGEA and HERA platforms. Subsequently, the system deployment in a real scenario is depicted. Finally, the conclusions are presented.

\section{Background and Problem Description}

Ageing is a natural evolution among all living things, part of the life cycle, well known by everyone. This life cycle can stretch based on several surrounding factors, among them, food diet, living habits, social aspects, society, and medication supplements. Nowadays, there is an ever growing need to supply constant care and support to the elderly and the drive to find more effective ways to provide such care has become a major challenge for the scientific community. During the last three decades the number of Europeans over 60 years old has risen by about $50 \%$. Today they represent more than $25 \%$ of the population and it is estimated that in 20 years this percentage will rise to one third of the population, meaning 100 millions of 
citizens [8]. In the USA, people over 65 years old are the fastest growing segment of the population [9] and it is expected that in 2020 they will represent about 1 of 6 citizens totaling 69 million by 2030. Furthermore, over $20 \%$ of people over 85 years old have a limited capacity for independent living, requiring continuous monitoring and daily care. Some estimations of the World Health Organization show that in 2025 there will be more than 1000 million people aged over 60 in the world, so if this trend continues, by 2050 will be double, with about the $80 \%$ concentrated in developed countries [1]. Life expectancy increase over time, societies, demographic trends, which demands governments worldwide to take proper measures towards ageing challenges. As the evolution theory indicates, made by natural selection and environmental adaptation are key for surviving, it is possible to transpose the same ideas into the care domain, where ICT is "naturally" be incorporated and blended inside care environments.

With the appearance of AmI and AAL based systems, one of the most benefited segments of population will be the elderly and people with disabilities [2]. It will improve important aspects of their life, especially health care. The elderly of the future will have potential for an autonomous and active lifestyle, including all aspects of a healthy and fulfilled life such as sports, nutrition, education, entertainment, travel and social interaction. AmI provides a framework for developing intelligent environments that can assist the elder in the daily life [9]. More specifically, ambient assisted living (AAL) focus on the positive, as well as the negative aspects of growing life spans and improving the existing private and professional networks and infrastructures, thus reducing the burden on caregivers and increasing the autonomy of the caretakers at the same time, while preserving the immense skills and experiences of the elderly cohort within our society. In particular, AAL can be used to improve the daily life in a geriatric residence or in the elder's home.

As can be observed in the revised works, sensing is a key factor in the development of AmI and AAL based systems. The data obtained from sensors provides the basis to make decisions about the actions that need to be taken by the caregivers or the automated intelligent system. Identification and location data, amongst others, play a prevalent role in most of the existing systems, and this kind of data is obtained by means of technology that is embedded, non-invasive and transparent for users. In this regard, users' locations given by Real-Time Locating Systems represent key context information to adapt systems to people's needs and preferences. Real-Time Locating Systems can be categorized by the kind of its wireless sensor infrastructure and by the locating techniques used to calculate the position of the tags (i.e., the locating engine). This way, there is a combination of several wireless technologies, such as RFID, WiFi, UWB and ZigBee, and also a wide range of locating techniques that can be used to determine the position of the tags. Among the most widely used locating techniques we have signpost, fingerprinting, triangulation, trilateration and multilateration [10].

A widespread technology used in Real-Time Locating Systems is Radio Frequency IDentification (RFID) [11]. In this case, the RFID readers act as exciters transmitting continuously a radio frequency signal that is collected by the RFID tags, which in turn respond to the readers by sending their identification numbers. In these kinds of locating systems, each reader covers a certain zone through its radio frequency signal, 
known as reading field. When a tag passes through the reading field of the reader, it is said that the tag is in that zone. Locating systems based on Wireless Fidelity (Wi-Fi) take advantage of Wi-Fi WLANs (Wireless Local Area Networks) working in the 2.4GHz and 5.8GHz ISM (Industrial, Scientific and Medical) bands to calculate the positions of the mobile devices (i.e., tags) [12]. A wide range of locating techniques, then, can be used for processing the Wi-Fi signals and determining the position of the tags, including signpost, fingerprinting or trilateration. However, locating systems based on Wi-Fi present some problems such as the interferences with existing data transmissions and the high power consumption by the Wi-Fi tags. Ultra-Wide Band (UWB) is a technology which has been recently introduced to develop these kinds of systems. As it works at high frequencies (the band covers from $3.1 \mathrm{GHz}$ to $10.6 \mathrm{GHz}$ in the USA) [13], it allows to achieve very accurate location estimations. However, at such frequencies the electromagnetic waves suffer a great attenuation by objects (e.g., walls) so its use in indoor RTLS systems presents important problems, especially due to reflection and multipath effects. ZigBee is another interesting technology to build RTLSs. The ZigBee standard is specially intended to implement Wireless Sensor Networks and, as Wi-Fi, can work in the $2.4 \mathrm{GHz}$ ISM band, but also can work on the 868-915MHz band. Different locating techniques based on RSSI and LQI can be used on ZigBee WSNs (e.g., signpost or trilateration). Moreover, it allows building networks or more than 65,000 nodes in star, cluster-tree and mesh topologies [10]. ZigBee is, indeed, the wireless technology selected for our research.

From this point of view, it is important to assist the caregiver and the caretaker in the daily routine which, commonly, is composed of a series of tasks. Lanzola et al. [14], for instance, presents a methodology that facilitates medical applications and proposes a generic computational model for its implementation. Such a model could be specialized to manage all kind of information and knowledge in a hospital environment. However, the method proposed by Lanzola et al. [14] is very abstract and does not take into account the possibility of modeling certain technologies common in hospital environments (as shown in this paper) as wireless or RFID. Others such as Decker and Li [15], propose a system to increase hospital efficiency using global planning and scheduling techniques. However, this system does not work on wireless devices and does not use location information or RFID technology.

As observed in this section, the elder of the future require innovative systems to improve different aspects of his life, and both technology and intelligent systems are key factors in this process. There exist different projects focused on improving the quality of life. However, they do not take into account the use of wireless devices with hardware-embedded reactive agents platforms running on them. This paper proposes an innovative solution based on agent technology, a set of wireless technologies and indoor real-time locating techniques.

\section{System Overview}

This section describes the main features of the multi-agent system proposed in this paper. It is an Ambient Intelligence based multi-agent system aimed at improving 
healthcare of dependent people at the geriatric residences and their own homes. An essential aspect in this system is the use of Wireless Sensor Networks (WSN) to provide the agents with automatic and real-time information of the environment and allow them to react upon it. This way, the system makes use of several WSNs in order to gather context information in an automatic and ubiquitous way. The system enables an extensive integration of WSNs and provides a greater simplicity of deployment, thus optimizing the reutilization of the available resources in such networks. The agents in the system are implemented using two different agent platforms, PANGEA (Platform for Automatic coNstruction of orGanizations of intElligents Agents) [6] and HERA (Hardware-Embedded Reactive Agents) [7].

On the one hand, PANGEA is an agent platform to develop open multi-agent systems, specifically those including organizational aspects such as virtual agent organizations. The platform allows the integral management of organizations and offers tools to the end user. Additionally, it includes a communication protocol based on the IRC standard, which facilitates implementation and remains robust even with a large number of connections. The dynamics of open environments is one of the reasons that have encouraged the use of Virtual Organizations of Agents (VOs) [6]. A $\mathrm{VO}$ is an open system designed for grouping; it allows for the collaboration of heterogeneous entities and provides a separation between the form and function that define their behavior.

On the other hand, HERA [7] is an evolution of SYLPH (Services laYers over Light PHysical devices) [16]. SYLPH has the ability to use dynamic and selfadaptable heterogeneous WSNs. In HERA, agents are directly embedded into wireless nodes. This way, through the integration of HERA and PANGEA there is no difference between a software and a hardware agent. That is, PANGEA provides a high-level management system, where organizational aspects are taken into account, regardless of whether the agent is a piece of code or a wireless sensor. PANGEA includes facilities for implementing VOs and suborganizations, following any topology and with the appropriate tools for managing the VO itself; it is a complete platform for the execution and management of VOs, and incorporates intelligent agents with advances capacities for learning and adaptation.

\subsection{The PANGEA Agents Platform}

PANGEA is a platform that can integrally create, manage and control VOs. PANGEA manages roles, norms and the organizations that facilitate the inclusion of organizational aspects. The services are also included completely separated from the agent, facilitating their flexibility and adaption. PANGEA incorporates a CBR-BDI reasoning mechanism available for the agents. The basic agent types defined in PANGEA are:

- OrganizationManager: the agent responsible for the actual management of organizations and suborganizations. It is responsible for verifying the entry and exit of agents, and for assigning roles. To carry out these tasks, it works with the OrganizationAgent, which is a specialized version of this agent. 
- InformationAgent: the agent responsible for accessing the database containing all pertinent system information.

- ServiceAgent: the agent responsible for recording and controlling the operation of services offered by the agents. It works as the Directory Facilitator defined in the FIPA standar.

- NormAgent: the agent that ensures compliance with all the refined norms in the organization.

- CommunicationAgent: the agent responsible for controlling communication amongst agents, and for recording the interaction between agents and organizations.

- SnifferAgent: manages the message history and filters information by controlling communication initiated by queries.

We have created a service-oriented platform that can take maximum advantage of the distribution of resources. To this end, all services are implemented as Web Services. This makes it possible for the platform to include both a service provider agent and a consumer agent, thus emulating a client-server architecture. The provider agent (a general agent that provides a service) knows how to contact the web service, the rest of the agents know how to contact with the provider agent due to their communication with the ServiceAgent, which contains this information about services. Once the client agent's request has been received, the provider agent extracts the required parameters and establishes contact. Once received, the results are sent to the client agent. Using Web Services also allows the platform to introduce the SOA architecture (Service-Oriented Architecture) into MAS systems. SOA is an architectural style for building applications that use services available in a network such as the web [17]. It promotes loose coupling between software components so that they can be reused. Applications in SOA are built based on services. A service is an implementation of a well-defined functionality, and such services can then be consumed by clients in different applications or processes. SOA allows for the reuse of existing services and a level of flexibility that was not possible before.

Each suborganization or work unit is automatically provided with an OrganizationAgent by the platform during the creation of the suborganization. This OrganizationAgent is similar to the OrganizationManager, but is only responsible for controlling the suborganizationn, and can communicate with the OrganizationManager if needed. If another suborganization is created hierarchically within the previous suborganization, it will include a separate OrganizationAgent that communicates with the OrganizationAgent from the parent organization. These agents are distributed hierarchically in order to free the OrganizationManager of tasks. This allows each OrganizationAgent to be responsible for a suborganization although, to a certain extent, the OrganizationManager can always access information from all of the organizations. Each agent belongs to one suborganization and can only communicate with the OrganizationAgent from its own organization; this makes it possible to include large suborganizational structures without overloading the AgentManager. All of the OrganizationAgents from the same level can communicate with each other, unless a specific standard is created to prevent this. 


\subsection{The HERA Agents Platform Running on the Wireless Sensor Nodes}

This section briefly describes the HERA (Hardware-Embedded Reactive Agents) platform [7] and the WSN-SOA platform on which it is based, SYLPH (Services laYers over Light PHysical devices) [16]. In HERA agents are directly embedded on the WSN nodes and their services can be invoked from other nodes in the same network or other network connected to the former one. HERA is an evolution of the SYLPH platform [16]. SYLPH follows a SOA model [17] for integrating heterogeneous WSNs in AmI-based systems. HERA takes a step ahead over SYLPH, embedding agents directly on the wireless nodes. SYLPH implements an organization based on a stack of layers. Each layer in one node communicates with its peer in another node through an established protocol. In addition, each layer offers specific functionalities to the immediately upper layer in the stack.

The SYLPH Message Layer (SML) offers the upper layers the possibility of sending asynchronous messages between two nodes through the SYLPH Services Protocol (SSP), the internetworking protocol of the SYLPH platform. The SYLPH Application Layer (SAL) allows different nodes to directly communicate with each other using SSDL (SYLPH Services Definition Language) requests and responses that will be delivered in encapsulated SML messages following the SSP. The SSDL is the IDL (Interface Definition Language) used by SYLPH. SSDL has been specifically designed to work with limited computational resources nodes [16]. Furthermore, there are other interlayer services offered by the SAL for registering services or finding services offered by other nodes. In fact, these interlayer services call other interlayer services offered by the SYLPH Services Directory Sub-layer (SSDS). Any node that stores and maintains services tables is called SYLPH Directory Node (SDN). In SYLPH, a node in a specific type of WSN (e.g., ZigBee) can directly communicate with a node in another type of WSN (e.g., Bluetooth). Therefore, several heterogeneous WSNs can be interconnected through a SYLPH Gateway. A SYLPH Gateway is a device with several hardware network interfaces, each of which is connected to a distinct WSN. The SYLPH Gateway stores routing tables for forwarding SSP packets amongst the different WSNs with which it is interconnected.

The HERA platform adds its own agents layer over the SYLPH stack. This way, HERA agents running on WSNs with different radio technology can communicate amongst them through one or more SYLPH Gateways [16]. The main components added by HERA to the SYLPH stack are the HERA Agents Layer (or just HERA) and the HERA Communication Language Emphasized to Simplicity (HERACLES). HERA agents are specifically intended to run on devices with reduced resources, similar as SYLPH was designed for. Each HERA agent is an intelligent piece of code running over the SAL. There must be almost one facilitator agent in every agent platform. This agent is the first created in the platform and acts as a directory for searching agents. In HERA, the equivalent of this agents are the HERA-SDNs (HERA Spanned Directory Nodes). HERA agents communicate amongst them through HERACLES, the agent communication language designed for being used under HERA. 


\section{Case Study}

This section presents a real scenario where the system has been deployed to provide healthcare for elderly people. The scenario consists of a telemonitoring system for elderly at a geriatric residence where elderly can be monitored not only at the residence, but also at their own homes. This way, the daily life and the safety of the external patients are also improved, delaying or even avoiding the moment in which they need to move to residence. The name of the residence have been omitted due to confidentiality reasons.

The system includes an innovative indoor and outdoor Real-Time Locating System that features an outstanding precision, flexibility and automation integration [18] [19]. Moreover, the application includes home automation capabilities, so that a light sensor can make a lamp to be switched on or dimmed by means of the invocation or a certain service stored in a wireless actuator node connected to the relay or dimmer of the respective lamp. Agents in the system collaborate with context-aware agents that employ Wireless Sensor Networks and automation devices to provide automatic and real-time information about the environment, and allow users to interact with their surroundings, controlling and managing physical services (i.e., heating, lights, switches, etc.). All hardware is someway integrated with agents, providing automatic and real-time information about the environment that is processed by the agents to automate tasks and manage multiple services.

On the one hand, there is a locating infrastructure at the geriatric residence for positioning patients, medical personnel and assets within the building. The configuration used in the system (shown in Figure 1) consists of a ZigBee tag mounted on a bracelet worn on the users' wrist or ankle, several ZigBee readers installed over protected zones, and a central workstation where all the information is processed and stored. These readers are installed all over the facilities so that the system can detect when a user was trying to enter a forbidden area according to the user's permissions. The ZigBee network also allows obtaining information of the environment from different sensors, such as temperature sensors, light sensors, as well as smoke and gas detectors. Each ZigBee node includes an 8-bit RISC (Atmel ATmega 1281) microcontroller with $8 \mathrm{~KB}$ of RAM, $4 \mathrm{~KB}$ of EEPROM and $128 \mathrm{~KB}$ of Flash memory and an IEEE $802.15 .4 / \mathrm{ZigBee} 2.4 \mathrm{GHz}$ transceiver (Atmel AT86RF230). These devices have several communication ports (GPIO, ADC, I2C and UART through USB or DB-9 RS-232) to connect to distinct devices, including a wide range of sensors and actuators [18]. In addition, it can be used different locating techniques using these devices as readers and also as tags carried by patients and medical personnel. These devices are small enough to be carried by a patient, a caregiver or even an object, and provide a battery life of up to six months. The location of users is given as coordinated points obtained from the locating techniques provided by a locating engine [19]. All information obtained by means of these technologies is processed by the PANGEA agents. Depending on the system requirements, several interfaces can be executed. The interfaces show basic information about nurses and patients (name, tasks that must be accomplished, location inside the residence, etc.) and the building (specific room temperature, lights 
status, etc.). The system allows users to keep track of any tag in the system as well as receive distinct alerts in real-time coming from the system in any Web-based device, such as PC or a smartphone carried by doctors and nurses. Among the different alerts there are panic button alerts (when a user press a panic button on its tag or in a fixed device including such a button), forbidden area alerts (when a user enter into a forbidden area according to its permissions), as well as low-battery alerts (if some tag in the system should be recharged).

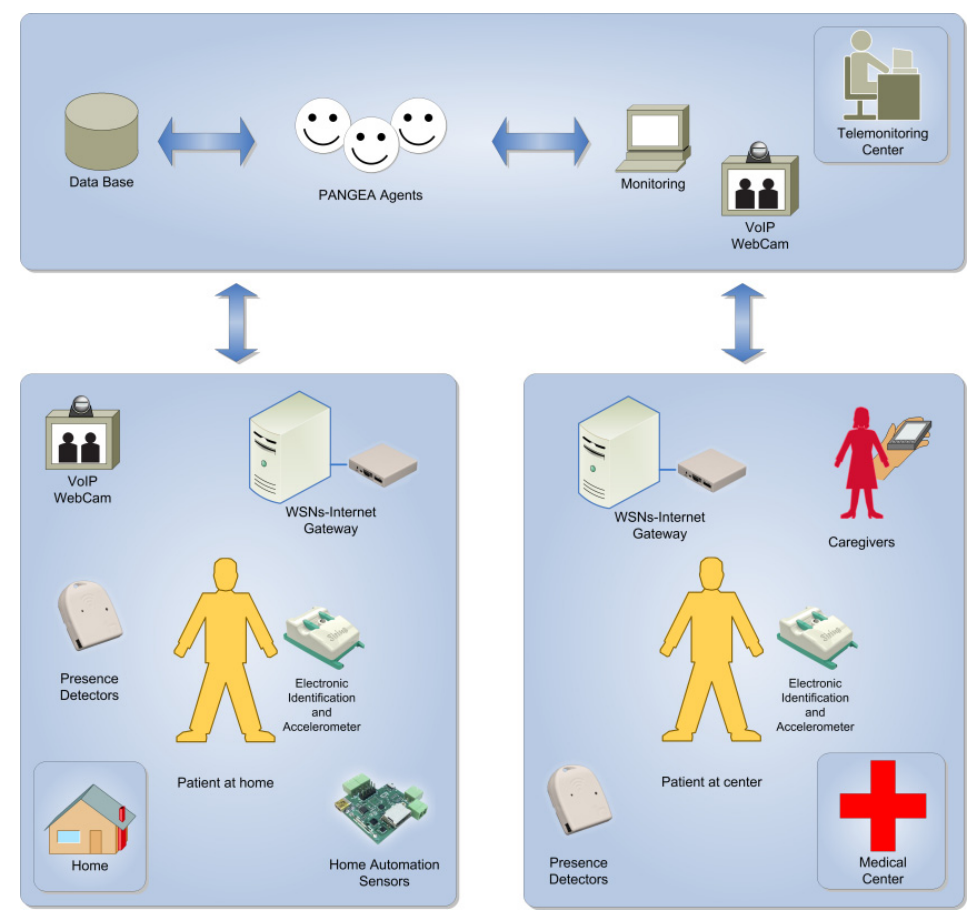

Fig. 1. Communication and infrastructure schema of the telemonitoring system

The ZigBee infrastructure has been deployed in a $600 \mathrm{~m} 2$ area within the residence where live dependent people with distinct dementias such as Alzheimer's disease. The locating infrastructure is intended to provide the real-time position of people (i.e., patients and medical personnel) and assets (i.e., wheelchairs and lifters) with an average accuracy of 2 meters within the monitored area. Figure 2 shows one of the Web-based interfaces of the telemonitoring system in the monitored area. The infrastructure deployed for the first pilot in the $600 \mathrm{~m} 2$ area includes 64 fixed ZigBee nodes [18] acting as readers and collecting nodes, 15 tags for residents and nurses, as well as a location server running in a local computer.

On the other hand, there is deployed a home automation in each patient's home for telemonitoring the environment where he or she lives from a remote control center installed in the own geriatric residence. Thus, there is a computer connected to a remote healthcare telemonitoring center (i.e. the geriatric residence) via Internet. 
Alerts can be forwarded from the patients' homes to the caregivers in the remote center, allowing them to communicate with patients in order to check the possible incidences. These alerts can be, for instance, the detection of a patient's fall or a high smoke level in the patient's home. This computer acts as a ZigBee master node through a physical wireless interface (e.g., a ZigBee network adapter as a ZigBee USB dongle or a ZigBee node connected through the computer's USB port) [18]. If, for instance, the monitored patient falls over on the floor, his fall detector gets from its accelerometer a measurement higher than a previously specified threshold. This sensor (i.e. accelerometer) invokes a service stored in the Internet-HERA Gateway. Such service initiates a VoIP and webcam connection to be established between the telemonitoring center and the patient's home through Internet. This way, caregivers in the remote center can watch the patient's home and talk with him in order to verify the accident. As the telemonitoring center accesses directly to a patients database, caregivers can see his medical data and home address. If the accident is confirmed, the caregivers send an ambulance to his home.

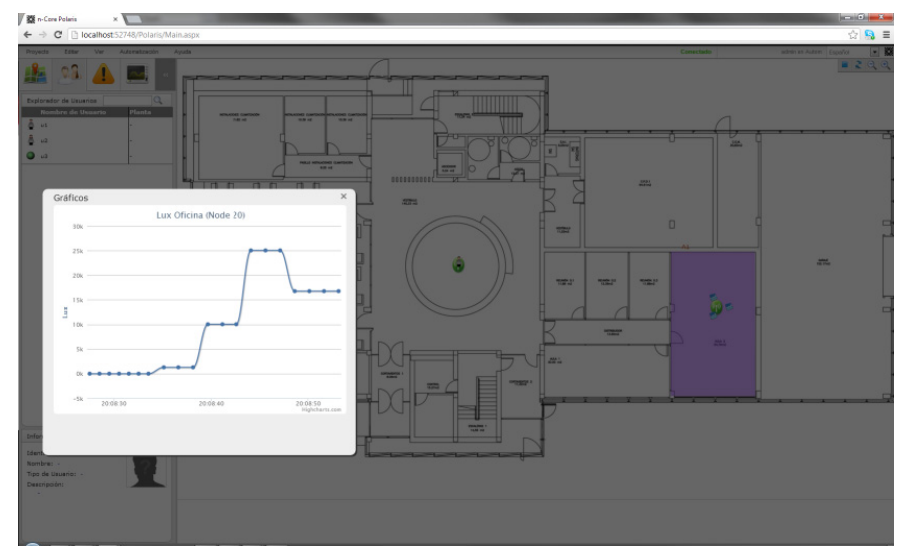

Fig. 2. Web-based interface for tracking people and assets

The home automation infrastructure for the remote telemonitoring of the elderly at their homes is formed by a ZigBee wireless sensor network fully integrated on the environment. For the pilot of the system, this network has been deployed in a typical bedroom in the own residence (simulating the patient's home), as shown in Figure 3.

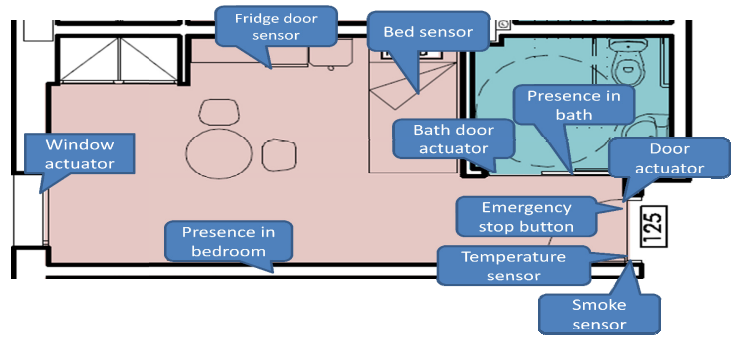

Fig. 3. Automation infrastructure deployed 
The automation infrastructure is formed by 16 sensors and actuators distributed in $5 \mathrm{ZigBee}$ nodes. The ZigBee nodes gather periodically the measurements from the sensors and forward this information to the remote center through one of the ZigBee nodes that acts as coordinator in the network and as gateway from the ZigBee network and the Internet.

\section{Conclusions}

In the future, health care for hospital patients, the elderly and people with other disabilities will require the use of new technologies that allow medical personnel to carry out their tasks more efficiently. In addition, the use of WSN and RTLS provides a high level of interaction among users and patients through the system and is fundamental in the construction of the intelligent environment.

The telemonitoring system presented in this paper improves security at home to dependents. It implements monitoring and alerting subsystems, as well as additional services to automatically react to emergency situations. The telemonitoring system's architecture goes a step ahead in designing systems for home care by offering features that make it easily adaptable to any pervasive environment. Unlike other telemonitoring systems, the presented system allows to integrate heterogeneous sensor networks from different technologies, even wired ones. So, it is possible to join multiple sensors in the same telemonitoring system using the technology that better fits each sensor's data characteristics. Furthermore, as result of the dynamic features of the PANGEA and HERA platforms, the presented system can be easily enhanced with new sensors without be redeployed and it can be adapted to new scenarios as even whole medical centers with many patients, whilst other approaches cannot. Finally, the system incorporates intuitive interfaces which facilitate usability and automatic adaptation to the user profile.

Acknowledgments. This project has been supported by the Spanish Ministry of Science and Technology project TIN 2009-13839-C03-03: Organizaciones Virtuales Adaptativas: Mecanismos, Arquitecturas y Herramientas (OVAMAH).

\section{References}

1. WHO: Global Age-friendly Cities: A Guide. World Health Organization (2007)

2. Emiliani, P.L., Stephanidis, C.: Universal access to ambient intelligence environments: Opportunities and challenges for people with disabilities. IBM Systems Journal (2005)

3. Wooldridge, M., Jennings, N.R.: Agent Theories, Architectures, and Languages: A Survey. In: Wooldridge, M.J., Jennings, N.R. (eds.) ECAI 1994 and ATAL 1994. LNCS, vol. 890, pp. 1-39. Springer, Heidelberg (1995)

4. Sarangapani, J.: Wireless Ad hoc and Sensor Networks: Protocols, Performance, and Control, 1st edn. CRC (2007)

5. Nerguizian, C., Despins, C., Affès, S.: Indoor Geolocation with Received Signal Strength Fingerprinting Technique and Neural Networks. In: de Souza, J.N., Dini, P., Lorenz, P. (eds.) ICT 2004. LNCS, vol. 3124, pp. 866-875. Springer, Heidelberg (2004) 
6. Zato, C., Villarrubia, G., Sánchez, A., Barri, I., Rubión, E., Fernández, A., Rebate, C., Cabo, J.A., Álamos, T., Sanz, J., Seco, J., Bajo, J., Corchado, J.M.: PANGEA - Platform for Automatic coNstruction of orGanizations of intElligent Agents. In: Omatu, S., Paz Santana, J.F., González, S.R., Molina, J.M., Bernardos, A.M., Rodríguez, J.M.C. (eds.) Distributed Computing and Artificial Intelligence. AISC, vol. 151, pp. 229-240. Springer, Heidelberg (2012)

7. Alonso, R.S., Tapia, D.I., Bajo, J., García, O., De Paz, J.F., Corchado, J.M.: Implementing a hardware-embedded reactive agents platform based on a service-oriented architecture over heterogeneous wireless sensor networks. Ad Hoc Networks 11(1), 151-166 (2013) ISSN 1570-8705, 10.1016/j.adhoc.2012.04.013

8. Camarinha-Matos, L., Afsarmanesh, H.: Design of a Virtual Community Infrastructure for Elderly Care. In: Camarinha-Matos, L.M. (ed.) Proceedings of PRO-VE 2002, Sesimbra, Portugal (2002)

9. Kohn, L.T., Corrigan, J.M., Donaldson, J.: To Err is human: Building a Safer Health System. Committee on Quality of Health Care in America Institute of Medicine. National Academy Press, Washington, DC (1999)

10. Liu, H., Darabi, H., Banerjee, P., Liu, J.: Survey of Wireless Indoor Positioning Techniques and Systems. IEEE Trans. Syst. Man Cybern. Part C-Appl. Rev. 37, 10671080 (2007)

11. Tapia, D.I., De Paz, J.F., Rodríguez, S., Bajo, J., Corchado, J.M.: Multi-Agent System for Security Control on Industrial Environments. International Transactions on System Science and Applications Journal 4(3), 222-226 (2008)

12. Ding, B., Chen, L., Chen, D., Yuan, H.: Application of RTLS in Warehouse Management Based on RFID and Wi-Fi. In: 4th International Conference on Wireless Communications, Networking and Mobile Computing, WiCOM 2008, pp. 1-5 (2008)

13. Stelios, M.A., Nick, A.D., Effie, M.T., et al.: An indoor localization platform for ambient assisted living using UWB. In: Proceedings of the 6th International Conference on Advances in Mobile Computing and Multimedia, pp. 178-182. ACM, Linz (2008)

14. Lanzola, G., Gatti, L., Falasconi, S., Stefanelli, M.: A Framework for Building Cooperative Software Agents in Medical Applications. Artificial Intelligence in Medicine 16(3), 223-249 (1999)

15. Decker, K., Li, J.: Coordinated Hospital Patient Scheduling. In: Proceedings of the Third International Conference on Multi Agent Systems, Paris, France, pp. 104-111 (1998)

16. Corchado, J.M., Bajo, J., Tapia, D.I., Abraham, A.: Using Heterogeneous Wireless Sensor Networks in a Telemonitoring System for Healthcare. IEEE Transactions on Information Technology in Biomedicine. Special Issue: Affective and Pervasive Computing for Healthcare 5518, 663-670 (2009)

17. Cerami, E.: Web Services Essentials: Distributed Applications with XML-RPC, SOAP, UDDI \& WSDL, 1st edn. O'Reilly Media, Inc. (2002)

18. Nebusens n-Core ${ }^{\circledR}:$ A Faster and Easier Way to Create Wireless Sensor Networks, http: / / www.n-core. info (accessed November 2012)

19. de Paz, J.F., Tapia, D.I., Alonso, R.S., Pinzón, C.I., Bajo, J., Corchado, J.M.: Mitigation of the ground reflection effect in real-time locating systems based on wireless sensor networks by using artificial neural networks. Knowl. Inf. Syst. 34, 193-217 (2013) 